\title{
Metastasis-free survival as a new endpoint in castration- -resistant prostate cancer
}

\author{
Kuzma $\mathbf{M}^{1}$, Kliment $\mathbf{J}^{2}$ \\ Jessenius Faculty of Medicine in Martin, Comenius University in Bratislava, Slovakia. \\ jan.kliment1@uniba.sk
}

\begin{abstract}
INTRODUCTION: Recent breakthrough recognition of metastasis-free survival as a clinically relevant endpoint has opened a new era in the management of advanced prostate cancer. The need for new, intermediate endpoints is the logical consequence of scientific advances in prostate carcinoma. The treatment algorithms for non-metastatic castration-resistant prostate cancer (M0 CRPC) have recently been updated by adding novel anti-androgen apalutamide, and also enzalutamide for high-risk patients.

OBJECTIVE: To review clinical evidence of metastasis-free survival as an efficacy endpoint used in prostate cancer studies, especially those in an advanced stage of the disease and identify its clinical benefit and correlation with overall survival.

METHODS: Literature search up to October 2019 was conducted, including clinical trials and clinical practice guidelines.

EVIDENCE SYNTHESIS: Metastasis-free survival (MFS) was used as the primary endpoint in the registration of clinical trials of second-generation anti-androgens. The study results have demonstrated the beneficial effect of these anti-androgens on delaying the development of metastases or death (MFS), with median MFS extended by 22-24 months. The correlation tests have shown a positive correlation of MFS and overall survival. CONCLUSION: The metastasis-free survival can be considered a clinically significant and reliable efficacy endpoint in both localized prostate cancer patients and MO CRPC patients being at high-risk of disease progression (Ref. 15). Text in PDF www.elis.sk.

KEY WORDS: metastasis-free survival, non-metastatic castration-resistant prostate cancer, apalutamide, enzalutamide.
\end{abstract}

\section{Introduction}

Overall survival (OS) is the traditional primary efficacy endpoint in clinical studies of advanced prostate cancer. The effect of available treatment modalities on overall survival was studied in patients with advanced, mostly metastatic castration-sensitive, as well as castration-resistant disease, where patients' survival was shorter due to the advanced nature of the carcinoma and where, consequently, a substantial number of events (deaths) occurred, meeting the requirements for a statistical analysis in terms of validity and power. However, the scientific progress and introduction of novel drugs for the treatment of metastatic prostate cancer in clinical practice have helped achieve better overall treatment outcomes, including longer patient survival. With innovative products

${ }^{1}$ Jessenius Faculty of Medicine in Martin, Comenius University in Bratislava, Slovakia, and ${ }^{2}$ Clinic of Urology, Jessenius Faculty of Medicine in Martin and University Hospital Martin, Comenius University in Bratislava, Martin, Slovakia

Address for correspondence: J. Kliment, Prof, MD, PhD, Clinic of Urology, Jessenius Faculty of Medicine in Martin and University Hospital Martin, Comenius University in Bratislava, Kollarova 2, SK-036 01 Martin, Slovakia.

Phone: +421.43 .4203444$

Acknowledgements: This work was supported by grant APVV-15-0181. being studied in clinical trials at earlier stages of prostate cancer, the issue of relevant surrogate endpoints for overall survival has gained prominence. Metastasis-free survival (MFS) is usually defined in clinical trials as the time from enrolment in the study or from treatment initiation until the detection of generalized disease manifesting with the development of distant metastases, or until the patient's death. MFS as the primary endpoint was studied in patients with localized prostate cancer and also repeatedly in patients with non-metastatic CRPC, most recently in three clinical phase III registration studies with innovative anti-androgens. Prostate cancer progression and bone involvement usually lead to the onset of symptoms, greater subjective complaints and reduced quality of life. Metastatic disease is also associated with increased mortality $(1,2)$. This article is based on the method of literature search of studies evaluating metastasis-free survival (MFS) as an endpoint in prostate cancer. The PubMed database was searched for professional publications and clinical practice guidelines, using pre-defined keywords, including prostate cancer, non-metastatic, castrate resistance, metastasis-free survival.

\section{MFS in localized prostate cancer}

The adequacy of MFS as a surrogate efficacy endpoint for overall survival in patients with localized prostate cancer was 
411-414

evaluated in a meta-analysis examining the data from more than 12 thousand patients pooled from 19 clinical studies. This twolevel validation meta-analysis has demonstrated a strong correlation of metastasis-free survival (MFS) and overall survival (OS), with Kendall's correlation coefficient of 0.91 for MFS (R2 0.83; 95\% CI: $0.71-0.88$ ). The results of this meta-analysis confirmed that MFS is a strong surrogate endpoint for overall survival in patients with localized prostate cancer and that it is associated with a significant risk of death from prostate cancer (3).

\section{MFS in non-metastatic castration-resistant prostate cancer}

CRPC is defined by castrate serum testosterone $<50 \mathrm{ng} / \mathrm{dL}$ or $1.7 \mathrm{nmol} / \mathrm{L}$ plus biochemical progression: three consecutive rises in PSA one week apart resulting in two $50 \%$ increases over the nadir, and a PSA $>2 \mathrm{ng} / \mathrm{mL}$ (4). Additionally, in case of nonmetastatic CRPC, the findings on conventional imaging examinations, such as a CT scan or bone scintigraphy, are negative. MFS in patients with non-metastatic castration-resistant prostate cancer (M0 CRPC) was initially studied in trials with zoledronic acid, atrasentan and zibotentan. A study of atrasentan (a selective endothelin receptor antagonist) used the time to disease progression, defined as the time to metastasis, as its primary efficacy endpoint. Atrasentan prolonged the time to metastasis by a median of 93 days compared with the control placebo arm, however, the result was non-significant $(p=0.288)$ (5). The efficacy of zibotentan, another endothelin receptor antagonist, compared to placebo in patients with M0 CRPC was studied in the ENTHUSE clinical programme. Neither this study has demonstrated some benefit on extending the overall survival or progression-free survival (HR for PFS: 0.89; 95\% CI: $0.71-1.12, \mathrm{p}=0.330$ ). The study was terminated early, based on the negative results obtained from its interim analysis (6). Another study comparing the effect of zoledronic acid on the delay in time to metastasis or death vs placebo in patients with M0 CRPC was also terminated before completion of accrual after interim analyses demonstrated that the observed event rate was lower than expected (7). Nevertheless, the placebo group analysis (201 patients) served to evaluate the natural development of M0 CRPC in patients on androgen deprivation therapy. The primary endpoints of the study were the time to first bone metastasis, overall survival and bone metastasis-free survival (BMFS). It has been demonstrated that over a period of 2 years, $33 \%$ of M0 CRPC patients developed distant metastases, with median bone metastasis-free survival of 30 months. During the same period, 22 $\%$ of the patients died and $42 \%$ either developed a bone metastasis or died. An analysis of predictive factors has shown that both baseline PSA level and PSA velocity are independent predictors of the time to bone metastasis, overall survival, and metastasisfree survival (MFS) (7). The results of Smith's analysis suggest that the general M0 CRPC population is not homogeneous, the disease has an indolent course in some patients, and that patients at high risk of disease progression should be considered for studies of M0 CRPC.

Another study of M0 CRPC was designed for high-risk patients. The efficacy of denosumab in patients with non-metastatic
CRPC at high risk of disease progression (PSA $\geq 8,0 \mathrm{ng} / \mathrm{mL}$ and/ or PSA doubling time $\leq 10,0$ months) was studied in a phase III randomized study in which a total of 1,432 enrolled patients were randomized in a $1: 1$ ratio to receive either denosumab (716 patients) or placebo (716 patients). Bone metastasis-free survival (BMFS) was the primary endpoint of the study. BMFS was extended by a median of 4.2 months ( 29.5 months in the denosumab arm vs 25.2 months in the placebo arm), corresponding to a $15 \%$ reduction in the risk of bone metastases or death (HR 0.85; 95\% CI: $0.73-0.98, \mathrm{p}=0.028$ ). Denosumab also provided a significant delay in the time to first metastasis (HR 0.84; 95\% CI: 0.71-0.98, $\mathrm{p}=0.032$ ) (8). No difference in the overall survival has been demonstrated $(p=0.9)$. Despite the positive effect of denosumab on improved BMFS, the US Food and Drug Administration (FDA) assessed the benefit of denosumab as clinically insignificant and in 2012 rejected the application for its authorisation for the treatment of high-risk M0 CRPC (9).The results of the randomized studies described above, obtained from a total of over 4,000 patients, were negative, with no positive effect of the studied molecules on MFS, or with a positive but clinically insignificant benefit.

\section{MFS in non-metastatic castration-resistant prostate cancer in the new era of second-generation anti-androgens}

The first drug approved by the FDA on the basis of a demonstrated benefit on the examined primary endpoint MFS was apalutamide, a novel second-generation non-steroidal anti-androgen. Apalutamide is selective androgen receptor (AR) inhibitor that binds directly to the ligand-binding domain of AR. Apalutamide prevents AR nuclear translocation, inhibits DNA binding, impedes AR-mediated transcription, and lacks androgen receptor agonist activity (10). The efficacy and safety of apalutamide in patients with high-risk M0 CRPC was evaluated in an international, multicentre, double-blind, placebo-controlled phase III study (SPARTAN) which enrolled a total of 1,207 M0 CRPC patients who were randomized in a 2:1 ratio to receive either apalutamide $(240 \mathrm{mg}$ daily) plus androgen deprivation therapy ( 806 patients) or placebo plus ADT (401 patients). The patients enrolled in the study had a PSA doubling time (PSADT) of $\leq 10$ months, i.e. they were patients at high-risk of progression to metastatic disease and death of prostate cancer. The metastasis-free survival (MFS) primary endpoint of the study was defined as the time from randomization to the first detection of distant metastasis on imaging (as assessed by means of blinded independent central review) or death from any cause, whichever occurred first. The first interim analysis showed that apalutamide provided a significant improvement in MFS, while reducing the risk of developing distant metastases or death by $72 \%$ compared to placebo (HR=0.28; 95\% CI: $0.23-$ $0.35, \mathrm{p}<0.001)$. The median MFS was 40.5 months in the apalutamide arm vs 16.2 months in the placebo arm, thus providing a benefit of 24.3 months. The MFS benefit was consistent across the pre-defined patient subgroups and the benefit of apalutamide was also supported by positive results of the secondary and exploratory endpoints: progression-free survival, progression-free survival 2 (PFS2: progression on the first subsequent therapy for 
mCRPC), time to metastases, time to symptomatic progression, time to initiation of chemotherapy, time to PSA progression and PSA response (11).

The next drug to demonstrate a benefit on metastasis-free survival was enzalutamide. Based on the results of the PROSPER trial, the indication of enzalutamide was extended to include highrisk M0 CRPC. PROSPER enrolled a total of 1,401 patients with high-risk M0 CRPC (PSADT $\leq 10$ months) who were randomized in a 2:1 ratio to receive either enzalutamide (160 mg daily) (933 patients) or placebo (498 patients). MFS as the primary endpoint was defined as the time from randomization to radiographic progression or death from any cause within 112 days of study treatment discontinuation. Enzalutamide demonstrated a clinically significant $71 \%$ reduction in the risk of radiographic progression or death $(\mathrm{HR}=0.29 ; 95 \% \mathrm{CI}: 0.24-0.35 ; \mathrm{p}<0.001)$. The median MFS was 36.6 months in the enzalutamide arm compared to 14.7 months in the placebo arm. A consistent MFS benefit was observed across all the studied sub-groups. The benefit of enzalutamide in the treatment of M0 CRPC has also been demonstrated for the secondary endpoints: time to PSA progression, proportion of PSA responders, time to first use of a subsequent anti-neoplastic therapy, health-related quality of life (12).

Darolutamide is a novel non-steroidal anti-androgen with a high affinity for AR that inhibits AR nuclear translocation and reduces the activation of genes required for cell growth. The structure of darolutamide is different from that of enzalutamide and apalutamide, exhibiting antagonistic activity even in AR mutations (such as F877L) and a low level of blood-brain barrier penetration $(2,10)$. The efficacy and safety of darolutamide in patients with M0 CRPC was studied in an international, randomized, placebocontrolled phase III study (ARAMIS). A total of 1,509 high-risk M0 CRPC patients (PSADT $\leq 10)$ were randomized in a 2:1 ratio to receive either darolutamide (600 mg twice daily) plus ADT (955 patients) or placebo plus ADT (554 patients). The metastasis-free survival (MFS) was the primary endpoint of the study. The median MFS was 40.4 months in the darolutamide arm compared to 18.4 months in the placebo arm (HR: 0.41 ; 95\% CI: $0.34-0.50, \mathrm{p}<$ 0.001 ), translating into a $59 \%$ reduction in the risk of developing distant metastases or death. Darolutamide has also demonstrated a benefit on or a positive trend in the studied secondary and exploratory endpoints, including the time to pain progression, time to cytotoxic chemotherapy, time to first symptomatic skeletal event, progression-free survival, time to PSA progression, time to initiation of subsequent anti-neoplastic therapy (2).

Although the interim analyses for all the three new-generation anti-androgens (apalutamide, enzalutamide, darolutamide) did not provide any significant results in terms of overall survival prolongation, it should be noted that a sufficient number of events (deaths) had not yet occurred at the time of the interim analyses, and the data were immature, due to the generally short follow-up period $(2,11,12)$. Recently published 2 nd interim, pre-specified analysis of OS in the SPARTAN trial with 285 (67\% of required) OS events, showed that apalutamide was associated with an improved OS compared with placebo (HR 0.75; 95\% CI 0.59-0.96, $\mathrm{p}<0.0197)$, although the $\mathrm{p}$-value did not cross the prespecified
O'Brien-Fleming boundary of 0.0121 (13). The final OS analyses of all three novel inhibitors studies are awaited.

\section{Association of metastasis-free survival and overall survival}

The landmark analysis of the MFS endpoint in the SPARTAN trial, i.e. an analysis of MFS at specific time intervals, has shown that patients who developed metastases at months 6,9 , and 12 had a significantly shorter median OS compared to patients without metastases ( $\mathrm{p}<0.0001$ for all three time intervals). The correlation of MFS and overall survival (OS) was assessed by Spearman's and Fleischer's statistics. Spearman's correlation coefficient was 0.62 , at the statistical significance level of $p<0.0001$, indicating a significantly positive correlation. Fleischer's correlation model also showed a positive correlation, with the coefficient of 0.69 . The correlation analyses of the SPARTAN trial with apalutamide have demonstrated a significant association of MFS and overall survival in patients with high-risk M0 CRPC (14). The correlation of MFS and OS in real-world population was analysed in 87 patients with M0 CRPC with the median baseline PSA level of 10.4 and PSADT of 5.1 months. The median MFS was 44.1 months and the median OS was 83.7 months (since developing M0 CRPC). The correlation analysis from real-world practice, too, has confirmed a strong correlation of MFS and OS (Pearson's coefficient 0.85, p $<0.001$, Kendall's tau coefficient $0.632, \mathrm{p}<0.001)$. The group of patients with PSADT $\leq 10$ months ( 70 patients) had a significantly shorter MFS compared to patients with a longer PSA doubling time: 40.2 months vs 90.4 months ( $\mathrm{p}=0.001)$, respectively, as well as a shorter OS: 76.2 months vs 104.3 months ( $\mathrm{p}=0.008)$, respectively (15). Metastasis-free survival (MFS) reported in this real-world population analysis was longer compared to the results of randomized clinical studies of novel anti-androgens, where the median MFS in the control arms was around 1.5 years $(2,11,12)$. Along with the retrospective design and a small patient sample, this may potentially be due to the low frequency of use of imaging examinations in real-world clinical practice compared to the frequent use of CT and bone scans in randomized phase III clinical trials.

\section{Conclusion}

Second-generation anti-androgens: apalutamide, enzalutamide and darolutamide have demonstrated a significant positive effect on improved metastasis-free survival (MFS) in patients with non-metastatic castration resistant prostate cancer (M0 CRPC) who are at high risk of disease progression. This delay in time to disease progression and generalization provides a clinically significant therapeutic benefit for these patients. The correlation analyses of the SPARTAN trial, as well as the results from real-world population, have demonstrated a significant correlation of metastasis-free survival (MFS) and overall survival in high-risk M0 CRPC patients. In high-risk M0 CRPC patients, just as in patients with localized carcinoma, MFS can be considered a relevant and reliable efficacy endpoint and a strong predictor of overall survival. 
411-414

\section{References}

1. Gartrell BA, Saad F. Managing bone metastases and reducing skeletal related events in prostate cancer. Nat Rev Clin Oncol 2014; 11: 335-345.

2. Fizazi K, Shore N, Tammela TL et al. Darolutamide in Nonmetastatic, Castration-Resistant Prostate Cancer. N Engl J Med 2019. doi: 10.1056/ NEJMoa1815671.

3. Xie W, Regan MM, Buyse M et al. Metastasis-free survival is a strong surrogate of overall survival in localized prostate cancer. J Clin Oncol 2017; 35: 3097-3104.

4. Mottet N, Van den Bergh R.C.N., Briers E et al. EAU - ESTRO ESUR - SIOG Guidelines on Prostate Cancer 2019. Available at: https:// uroweb.org/guideline/prostate-cancer/\#1_4

5. Nelson JB, Love W, Chin JL et al. Phase 3, Randomized Controlled Trial of Atrasentan in Patients with Nonmetastatic Hormone-Refractory Prostate Cancer. Cancer 2008; 113 (9): 2478-2487. doi: 10.1002/cncr.23864.

6. Miller K, Moul JW, Gleave M et al. Phase III, randomized, placebocontrolled study of once-daily oral zibotentan (ZD4054) in patients with non-metastatic castration-resistant prostate cancer. Prostate Cancer Prostatic Dis 2013; 16 (2): 187-192. doi: 10.1038/pcan.2013.2.

7. Smith MF, Kabbinavar F, Saad F et al. Natural History of Rising Serum Prostate-Specific Antigen in Men with Castrate Nonmetastatic Prostate Cancer. J Clin Oncol 2005; 23 (13): 2918-2925.

8. Smith MR, Saad F, Coleman R et al. Denosumab and bone-metastasisfree survival in men with castration-resistant prostate cancer: results of a phase 3, randomised, placebo-controlled trial. Lancet. 2012; 379 (9810): 39-46. doi: 10.1016/S0140-6736(11)61226-9.
9. FDACenter for Drug Evaluation and Research. Oncologic Drugs Advisory Committee Meeting, February 8, 2012. Available at: https://wayback. archive-it.org/7993/20170403224017/https://www.fda.gov/AdvisoryCommittees/CommitteesMeetingMaterials/Drugs/OncologicDrugsAdvisoryCommittee/ucm285400.htm

10. Rathkopf DE, Scher HI. Apalutamide for the treatment of prostate cancer. Expert Rev Anticancer Ther 2018; 18 (9): 823-836. doi: 10.1080/14737140.2018.1503954.

11. Smith MR, Saad F, Chowdhury S et al. Apalutamide Treatment and Metastasis-free Survival in Prostate Cancer. N Engl J Med 2018; 378 : 1408-1418. doi: 10.1056/NEJMoa1715546.

12. Hussain M, Fizazi K, Saad F et al. Enzalutamide in Men with Nonmetastatic, Castration-Resistant Prostate Cancer. N Engl J Med 2018; 378 : 2465-2474. doi: 10.1056/NEJMoa1800536.

13. Small EJ, Saad F, Chowdhury S et al. Apalutamide and Overall Survival in Non-Metastatic Castration-Resistant Prostate Cancer. Ann Oncol 2019; 0: 1-8. doi: 10.1093/annonc/mdz397.

14. Smith MR, Mehra M, Nair S et al. Association of metastasis-free survival (MFS) and overall survival (OS) in nonmetastatic castration-resistant prostate cancer (nmCRPC). doi: 10.1200/JCO.2018.36.15_supp1.5032. J Clin Oncology 2018; 36 (Suppl 15): 5032-5032.

15. Gagnon R, Alimohamed NS, Batuyong E et al. Metastasis-free Survival as a Predictor of Overall Survival in Non-Metastatic CastrationResistant Prostate Cancer. doi: 10.1200/JCO.2019.37.7_suppl.201. J Clin Oncol 2019; 37 (Suppl 7): 201-201.

Received January 28, 2020. Accepted March 28, 2020. 